
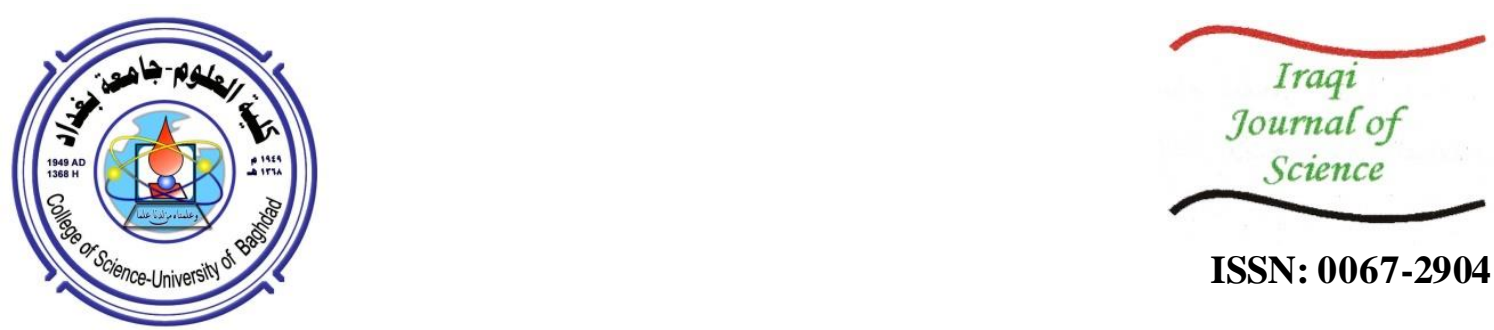

ISSN: 0067-2904

\title{
Splitting the one-Dimensional Wave Equation, Part II: Additional Data are Given by an End Displacement Measurement
}

\author{
Shilan Othman Hussein*1, M. S. Hussein ${ }^{2}$ \\ ${ }^{1}$ Department of Mathematics, College of Science, University of Sulaymaniyah, Sulaymaniyah, Iraq \\ ${ }^{2}$ Department of Mathematics, College of Science, University of Baghdad, Baghdad, Iraq
}

Received: $1 / 12 / 2019$

Accepted: $15 / 3 / 2020$

\begin{abstract}
In this research, an unknown space-dependent force function in the wave equation is studied. This is a natural continuation of [1] and chapter 2 of [2] and [3], where the finite difference method (FDM)/boundary element method (BEM), with the separation of variables method, were considered. Additional data are given by the one end displacement measurement $u_{x}(L, t) / u(L, t)$. Moreover, it is a continuation of [3], with exchanging the boundary conditionu $\mathrm{u}_{\mathrm{x}}(\mathrm{L}, \mathrm{t}) / \mathrm{u}(\mathrm{L}, \mathrm{t})$, where $\mathrm{x} \in[0, \mathrm{~L}]$ are extra data, by the initial condition $\mathrm{u}_{\mathrm{x}}(0, \mathrm{t}) / \mathrm{u}(0, \mathrm{t})$. This is an ill-posed inverse force problem for linear hyperbolic equation. Therefore, in order to stabilize the solution, a zeroth-order Tikhonov regularization method is provided. To assess the accuracy, the minimum error between exact and numerical solutions for the force is computed for various regularization parameters. Numerical results are presented and a good agreement was obtained for the exact and noisy data.
\end{abstract}

Keywords: Measure one end boundary condition; Finite difference method; Separation of variables method; Regularization; Inverse force problem.

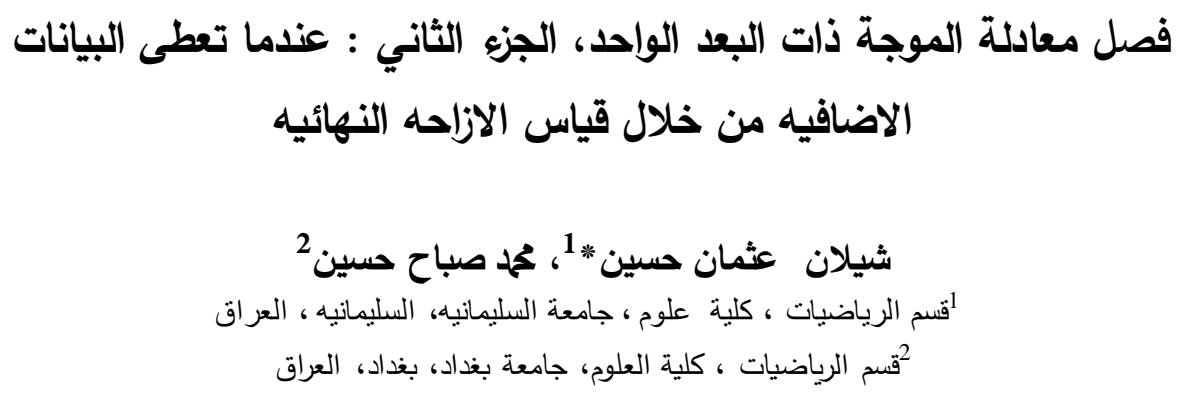




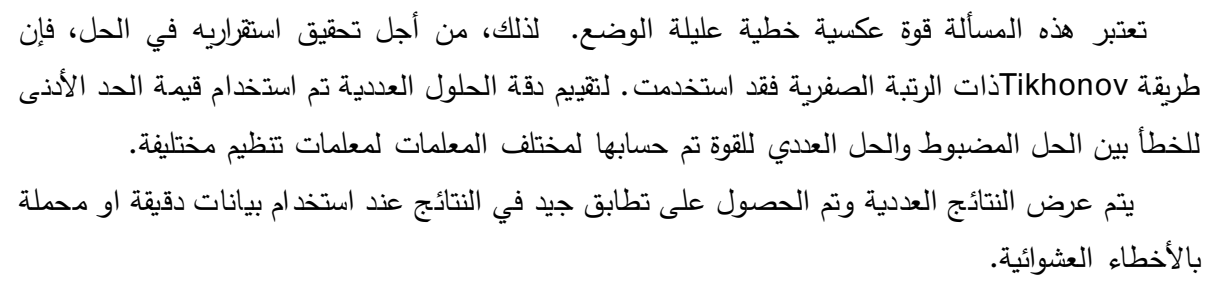

\section{Introduction}

An unknown force function in the wave equation can be experienced in many engineering applications dealing with wave, wind, seismic, explosion, or noise excitations [2, 4]. It can be found in physical problems as well; for instance, the vibrations of a spring or membrane, acoustic scattering, etc. The objective of this research is to provide the numerical solution for an inverse force problem for the nonhomogeneous hyperbolic equation, by considering the initial condition with boundary condition. Furthermore, in case of using Dirichlet boundary condition, the Neumann boundary conditions were taken as extra data. It is observed that we could also control the mixed data instead of the Dirichlet data. In a previous study [3], we used the finite difference method (FDM) to numerically discretize the wave equation with the method of separating the variables. Therefore, in order to extend the range of applicability, a different boundary condition has been applied in this study.

Similarly, as in [1-3], the resulting system of linear equations is ill-conditioned. Nearly, we obtained the same results (see Table 1, Table 2 and Figure 3 in [1]) and, for that reason, these tables and figures are omitted here. Consequently, we seek the Tikhonov regularization to regularize the solution.

This paper is organized as follows; Section 2 presents the mathematical formulation. Section 3 describes the numerical results and discussion. Section 4 includes the conclusions reached by this work.

\section{Mathe matical Formulations}

The required equation for a vibrating bounded structure $[0, L]$, acted upon by a force $f(x)$, is given by the wave equation $[1-3,5]$, as follows:

$$
\begin{gathered}
u_{t t}(x, t)=\nabla^{2} u(x, t)+f(x), \quad(x, t) \in(0, L) \times(0, T), \quad T>0, \\
u(x, 0)=u_{0}(x), \quad u_{t}(x, 0)=v_{0}(x), \quad x \in[0, L] \\
u(0, t)=p_{0}(t), \quad u(L, t)=p_{L}(t), \quad t \in[0, T],
\end{gathered}
$$

where $u(x, t), u_{0}$ and $v_{0}$ represent the displacement, the initial displacement and velocity, respectively. The above equation is a direct well-posed problem if $f(x)$ has been given, otherwise the problem becomes an inverse linear problem. Furthermore, in order to determine the pair solution $(u(x, t), f(x))$, we need to have extra conditions. For instance, (3) is a Dirichlet boundary condition, then an additional condition can be $u_{x}(L, t)$, namely:

$$
u_{x}(L, t)=q_{L}(t), \quad t \in(0, T)
$$

Also, we tried different conditions instead of the Dirichlet boundary condition (3) by using mixed boundary conditions.

$$
u(0, t)=p_{0}(t), \quad u_{x}(L, t)=q_{L}(t), \quad t \in(0, T),
$$

and in this case the additional condition has also changed to

$$
u(L, t)=p_{L}(t), \quad t \in(0, T) .
$$

By splitting equation (1) into $u=v+w[1-3,6]$, where $v$ satisfies the well-posed direct problem, we obtain

$$
\begin{aligned}
& v_{t t}(x, t)=v_{x x}(x, t), \quad(x, t) \in(0, L) \times(0, T), \\
& v(x, 0)=u_{0}(x), \quad v_{t}(x, 0)=v_{0}(x), \quad x \in[0, L], \\
& v(0, t)=p_{0}(t), \quad v(L, t)=p_{L}(t), \quad t \in(0, T) .
\end{aligned}
$$

Numerically, FDM has been used for solving (7)-(9) and to get $v_{x}(L, t)[2,3,5]$. In order to get $v(L, t)$, we will consider a different condition, i.e. using (5), namely the boundary condition (9) is changed to

$$
v(0, t)=p_{0}(t), \quad v_{x}(L, t)=q_{L}(t), \quad t \in(0, T) .
$$

The formula for the solution $v$ using FDM [2,7,5-8] is as follows:

$$
\begin{aligned}
& v_{i, j+1}=r^{2} v_{i+1, j}+2\left(1-r^{2}\right) v_{i, j}+r^{2} v_{i-1, j}-v_{i, j-1}, \quad i=\overline{1,(M-1)}, j=\overline{1,(N-1)}, \\
& v_{i, 0}=u_{0}\left(x_{i}\right), \quad i=\overline{0, M}, \quad \frac{v_{i, 1}-v_{i,-1}}{2 \Delta t}=v_{0}\left(x_{i}\right), \quad i=\overline{1,(M-1)}
\end{aligned}
$$




$$
v_{0, j}=P_{0}\left(t_{j}\right), \quad v_{M, j}=P_{L}\left(t_{j}\right), \quad j=\overline{0, N},
$$

By putting $j=0$ in equation (11) and using (12), we obtain [2, 3, 5-7]:

$$
\begin{gathered}
v_{i, 1}=\frac{1}{2} r^{2} u_{0}\left(x_{i+1}\right)+\left(1-r^{2}\right) u_{0}\left(x_{i}\right)+\frac{1}{2} r^{2} u_{0}\left(x_{i-1}\right)+(\Delta t) v_{0}\left(x_{i}\right), \quad i=\overline{1,(M-1) .} \\
-\frac{\partial v}{\partial x}\left(0, t_{j}\right)=-\frac{4 v_{1, j}-v_{2, j}-3 v_{0, j}}{2 \Delta x}, \frac{\partial v}{\partial x}\left(L, t_{j}\right)=\frac{3 v_{M, j}-4 v_{M-1, j}+v_{M-2, j}}{2 \Delta x}, \quad j=\overline{1, N} .
\end{gathered}
$$

where $v_{i, j}:=v\left(x_{i}, t_{j}\right), x_{i}=i \Delta x$, and $t_{j}=j \Delta t$, for $i=\overline{0, M}, j=\overline{0, N},\left(x_{i}, t_{j}\right)=(i \Delta x, j \Delta t)$, $\Delta x=\frac{L}{M}, t=\frac{T}{N}$, and $r=c \Delta t / \Delta x[2,3,5-7]$.

Also, let $(w, f)$ are the new variables satisfying the inverse problem [1-7], as follows:

$$
\begin{aligned}
& w_{t t}(x, t)=w_{x x}(x, t)+f(x), \quad(x, t) \in(0, L) \times(0, T), \\
& w(x, 0)=w_{t}(x, 0)=0, \quad x \in[0, L], \\
& w(0, t)=0, \quad w(L, t)=0, \quad t \in(0, T) . \\
& w_{x}(0, t)=q_{0}(t)-v_{x}(0, t), \quad t \in(0, T),
\end{aligned}
$$

But, for solving (16)-(19) numerically, we used the separation of variables in order to get $(w, f)$ [1-7]. In the case of changing the boundary condition (3) to condition (5), equations (18) and (19) are converted to

$$
\begin{aligned}
& w(0, t)=0, \quad w_{x}(L, t)=0, \quad t \in(0, T) \\
& w(L, t)=p_{L}(t)-v(L, t), \quad t \in(0, T)
\end{aligned}
$$

Based on the separation of variables [1-7] and by solving equations (16)-(19), we obtain

$$
\begin{gathered}
w_{k}(x, t ; \underline{b})=\frac{\sqrt{2}}{c^{2}} \sum_{k=1}^{K} \frac{b_{k}}{\lambda_{k}^{2}}\left(1-\cos \left(c \lambda_{k} t\right)\right) \sin \left(\lambda_{k} x\right), \quad(x, t) \in[0, L] \times[0, T] . \\
f_{K}(x)=\sqrt{2} \sum_{k=1}^{K} b_{k} \sin \left(\lambda_{k} x\right), \quad x \in(0, L),
\end{gathered}
$$

where $K$ is a truncation number and $\lambda_{k} \frac{k \pi}{L}$ for $k=\overline{1, K}$. The coefficient $\underline{b}=\left(b_{k}\right)_{k=\overline{1, K}}$ is to be calculated by imposing the additional boundary condition (19). These results in

$$
q_{L}(t)-v_{x}(L, t)=: g(t)=\frac{\partial w_{k}}{\partial x}(L, t ; \underline{b})=\frac{\sqrt{2}}{c^{2}} \sum_{k=1}^{K} \frac{b_{k}}{\lambda_{k}}\left(1-\cos \left(c \lambda_{k} t\right)\right) \cos \left(\lambda_{k} L\right), t \in[0, T] .
$$

such that $\underline{b}_{\lambda}=\left(Q^{t r} Q\right)^{-1} Q^{t r} \underline{g}$ where $Q_{n k}=\frac{\sqrt{2}\left(1-\cos \left(c \lambda_{k} t\right)\right) \cos \left(\lambda_{k} L\right)}{c^{2} \lambda_{k}}$.

For (16) and (17) with (20) and (21), we obtained the same $w_{K}(x, t ; \underline{b})$ in (22) and $f_{K}(x)$ in (23), but with different $\lambda_{k}$, where $\lambda_{k}=\left(k-\frac{1}{2}\right) \pi / L$ for $k=\overline{1, K}$. Also, $\underline{b}$ was determined by setting the additional condition (21), as follows

$$
p_{L}(t)-v(L, t)=: h(t)=\frac{\sqrt{2}}{c^{2}} \sum_{k=1}^{K} \frac{b_{k}}{\lambda_{k}^{2}}\left(1-\cos \left(c \lambda_{k} t\right)\right) \sin \left(\lambda_{k}\right), t \in[0, T] .
$$

In the same way, $\underline{b}_{\lambda}=\left(Q^{t r} Q\right)^{-1} Q^{t r} \underline{h}$ where $Q_{n k}=\frac{\sqrt{2}\left(1-\cos \left(c \lambda_{k} t\right)\right) \sin \left(\lambda_{k}\right)}{c^{2} \lambda_{k}^{2}}$.

For testing the stability, we add noisy data to $q_{L}(t)$ and $p_{L}(t)$, respectively, as follows

$$
q_{L}^{\epsilon}\left(t_{n}\right)\left|p_{L}^{\epsilon}\left(t_{n}\right)=q_{L}\left(t_{n}\right)\right| p_{L}\left(t_{n}\right)+\epsilon, \quad n=\overline{1, N},
$$

From a Gaussian normal distribution, $\epsilon$ can be determined where the mean zero and standard deviation $\sigma$ are given by [1-7]

$$
\sigma=p \% \times \max _{t \in[0, T]}\left|q_{L}(t)\right|_{\text {or }} p_{L}(t) \mid
$$

where $p \%$ represents the percentage of noise. The noisy data (26) also cause noise in $\left.g\right|_{\text {or }} h$ [1-3], as follows

$g^{\epsilon}\left(t_{n}\right)\left|h^{\epsilon}\left(t_{n}\right)=q_{L}^{\epsilon}\left(t_{n}\right)\right| p_{L}^{\epsilon}\left(t_{n}\right)-v_{x}\left(L, t_{n}\right)\left|v\left(L, t_{n}\right)=g\left(t_{n}\right)\right| h\left(t_{n}\right)+\epsilon, \quad n=\overline{1, N}$.

Finally, we apply the condition (24)|(25) with $\left.g\right|_{\text {or }} h$ replaced by $\left.g^{\epsilon}\right|_{o r} h^{\epsilon}$ in a least-squares penalised sense by minimizing the Tikhonov functional, which is in general a zeroth-order Tikhonov regularization solution (for more details, see [1-3]) to deal with stability,

$$
\underline{b}_{\lambda}=\left(Q^{t r} Q+\lambda I\right)^{-1} Q^{t r} \underline{g}^{\epsilon} \mid \underline{h}^{\epsilon},
$$

Note that, from equations (26)-(29), "|" means "or". 


\section{Numerical Results and Discussion}

In order to see how far the changing of boundary condition affects the accuracy of solution and to see the difference between boundary element method (BEM) and finite difference method (FDM), using the same example in [1-3], we have

$$
\begin{gathered}
u(x, t)=\sin (\pi x)+t+\frac{t^{2}}{2}, \quad f(x)=1+\pi^{2} \sin (\pi x), \quad x \in[0,1], \\
u(x, 0)=u_{0}(x)=\sin (\pi x), \quad u_{t}(x, 0)=v_{0}(x)=1, \quad x \in[0,1], \\
u(0, t)=p_{0}(t)=t+\frac{t^{2}}{2}, \quad u(1, t)=p_{L}(t)=t+\frac{t^{2}}{2}, \quad t \in(0,1],
\end{gathered}
$$

with the additional condition

$$
u_{x}(L, t)=q_{L}(t)=-\pi, \quad t \in[0,1]
$$

If the boundary condition is changed to

$$
u(0, t)=p_{0}(t)=t+\frac{t^{2}}{2}, \quad u_{x}(L, t)=q_{L}(t)=-\pi, \quad t \in[0,1]
$$

Then, in this case, the over-determination can be as follows:

$$
u(L, t)=p_{L}(t)=t+\frac{t^{2}}{2}, \quad t \in[0,1] .
$$

Figure 1 shows the numerical results for $v_{x}(L, t)$ obtained from (7)-(9) and $v(L, t)$ from (7), (8) and (10), both of which are obtained using the FDM (11)-(15) with $M=N \in\{20,40,80\}$. However, the exact solution for $\left(v_{x}(L, t), v(L, t)\right)$ does not exist, but from Figure 1, it can be seen that a convergent numerical solution is rapidly achieved. The value of $v_{x}(L, t)$ and $v(L, t)$ are then plugged into equations (24) and (25) to determine the values for $g\left(t_{n}\right)$ and $h\left(t_{n}\right)$, respectively. Also its noisy counterpart $g^{\epsilon}\left(t_{n}\right) \mid h^{\epsilon}\left(t_{n}\right)$ is given by (28) for $n=\overline{1, N},[1-3]$.

(a)

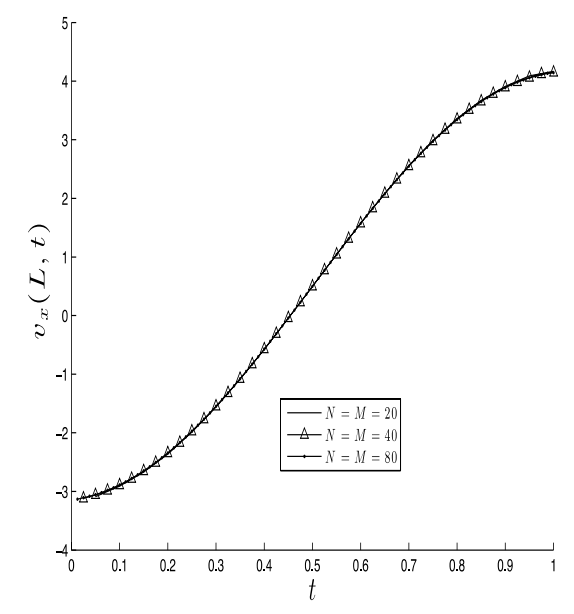

(b)

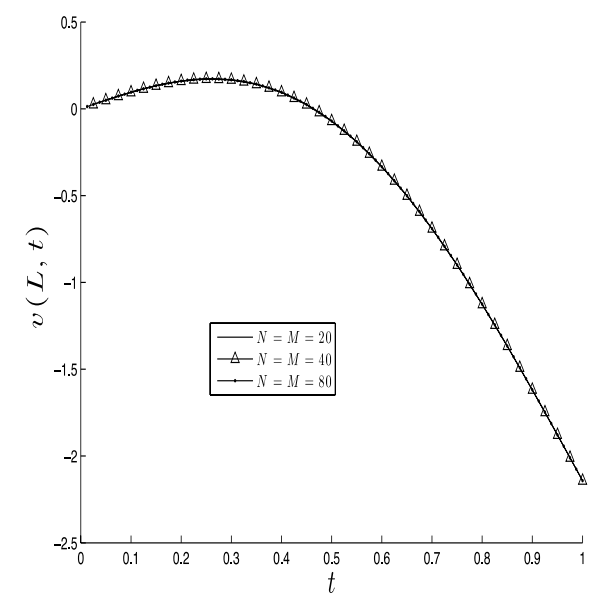

Figure 1-The numerical result for (a) $\mathbf{v}_{\mathbf{X}}(\mathbf{L}, \mathbf{t})$ (using Dirichlet boundary condition (9)) and (b) $\mathbf{v}(\mathbf{L}, \mathbf{t}$ ) (using mixed boundary condition (10)) are obtained using the FDM (11)-(15), with $\mathbf{M}=\mathbf{N} \in$ $\{20,40,80\}$.

In Figure-2(a), we note that the numerical solution of $\underline{b}$ from (24) was received for fixed $\mathrm{K}=$ $20, N=M=80$, when considering the exact data without noise (i.e. $\epsilon=0$ ) and then comparing with analytical values for the sine Fourier series coefficients $b_{k}=\sqrt{2} \int_{0}^{1} f(x) \sin (k \pi x) d x$ (see [1-3]). Also, when $f(x)$ is given by equation (30), one can see that a good approach was obtained between the numerical and exact values for $\underline{b}$. However, the method in [1] was changed to FDM and an additional condition $\mathrm{u}_{\mathrm{x}}(0, \mathrm{t})$ to condition $\mathrm{u}_{\mathrm{x}}(\mathrm{L}, \mathrm{t})$. Still, no different shape of the obtained figure can be seen; the reason is that the same exact values of $\underline{b}$ and the same boundary conditions $u(0, t)$ and $u(L, t)$ were used. 
Furthermore, Figure 2(b) shows the $\underline{b}=\left(b_{k}\right)_{k=\overline{1, K}}$ obtained from (25), in comparison with the exact sine Fourier series coefficients $b_{k}=\sqrt{2} \int_{0}^{1} f(x) \sin \left(\left(k-\frac{1}{2}\right) \pi x\right) d x$, when for $f(x)$ given by (30), there is a good agreement between the exact and numerical one, see Figure 3. Therefore, the method was changed to FDM instead of BEM, and there were different shapes of the plots obtained. We can still compare Figure-2 with Figures-(4 and 13) in [1]. In Figure-4, $\lambda_{k}=k \pi x$, but in here $\lambda_{k}=\left(k-\frac{1}{2}\right) \pi x$. Also, in Figure-13, cosine Fourier series is used instead of sine Fourier.

(a)

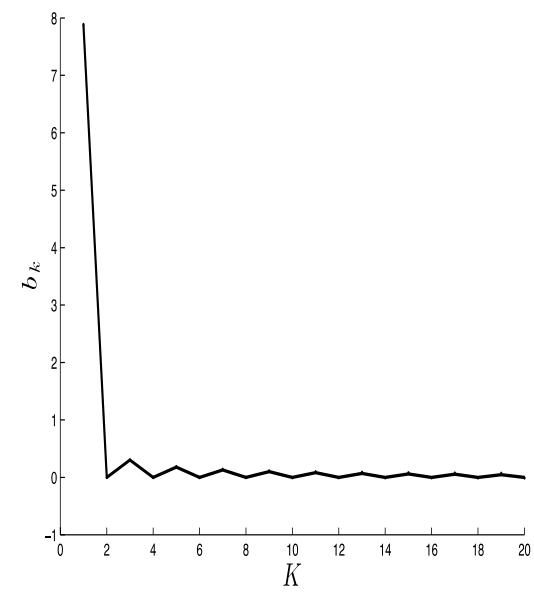

(b)

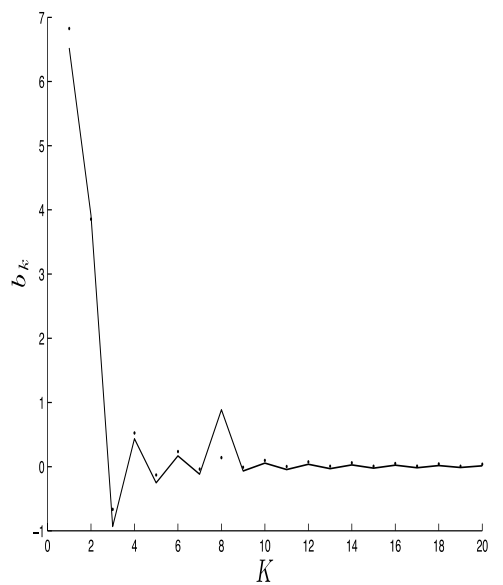

Figure 2-The numerical solution ( $\ldots)$ for $\left(b_{k}\right)_{k=\overline{1, K}}$ for $K=20, N=80$ obtained (a) from equation (24) in comparison with the exact solution $b_{k}=\sqrt{2} \int_{0}^{1} f(x) \sin (k \pi x) d x(-)$, (b) from equation (25) in comparison with the exact solution $b_{k}=\sqrt{2} \int_{0}^{1} f(x) \sin \left(\left(k-\frac{1}{2}\right) \pi x\right) d x(-)$.

Figure-3 shows a comparison between numerical (23) and exact values (30) of $f(x)$, where $k \in\{5,10,20\}$ and $N=M=80$. Numerical solution of $f(x)$ is earned after substituting numerical values $\underline{b}$ in equations (24) and (25). Moreover, Figure 3(a) represents the numerical and exact solutions for $f(x)$, where Dirichlet boundary condition (9) is applied, while Figure 3(b) represents these solutions when the mixed boundary condition (10) is used. These figures are obtained before adding noise to the exact data (i.e. $\epsilon=0$ ). It can be seen that accurate numerical solutions are achieved.

(a)

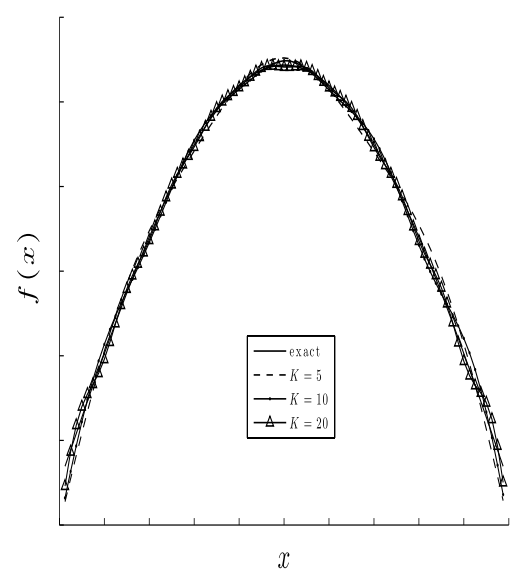

(b)

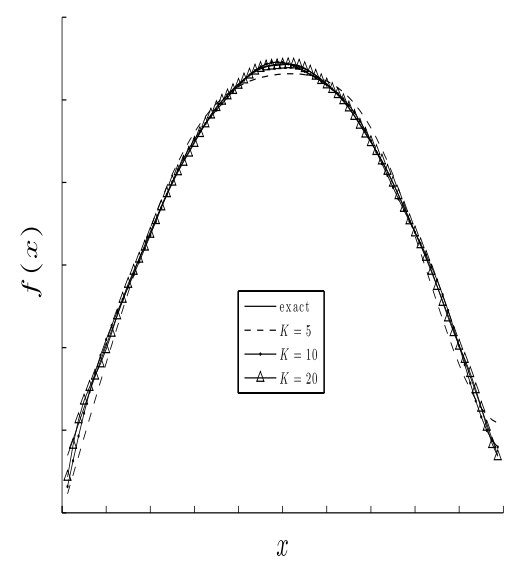

Figure 3-The exact solution (30) for $f(x)$ in comparison with the numerical solution (23), (a) Dirichlet boundary condition (9) is applied; (b) mixed boundary condition (10) is used for various $K \in\{5,10,20\}$, respectively.

After adding noise to the data (26), Figure 4 shows the unstable numerical solution for $f(x)$ with various $p \%$. In Figure 4(a), oscillations become highly unbounded, as $p \%$ increases for $f(x)$ when 
fixing $K=20$. In this case we selected $p \%=5 \%$. In addition, $p \%=1 \%$ and the various $K$ are shown in Figure 4(b). For performing regularization, using zeroth order Tikhonov regularization which yields the equation (29), various values of $\lambda$ such as $\lambda \in\left\{5 \times 10^{-4}, 10^{-4}, 5 \times 10^{-3}, 10^{-3}, \ldots, 10^{0}\right\}$ were tested. In order to choose the best $\lambda$, the error was calculated as ||$f_{\text {numerical }}-f_{\text {exact }}||=\sqrt{\sum_{n=1}^{N}\left(f_{\text {numerical }}\left(t_{n}\right)-f_{\text {exact }}\left(t_{n}\right)\right)^{2}}$ [4,6,7]. From Figure-5, it can be seen that the minimum error occurs around $\lambda=10^{-1}$ and $\lambda=5 \times 10^{-4}$, when it reaches the best approach for $f(x)$, as illustrated in Figure 6. Figure 5(a) and Figure 5(b) shows the minimum error around $\lambda=10^{-1}$ and $\lambda=5 \times 10^{-4}$, respectively. Meantime, a good approached value for numerical solution $f(x)$ can be seen in Figure-6(a) and Figure-6(b).

(a)

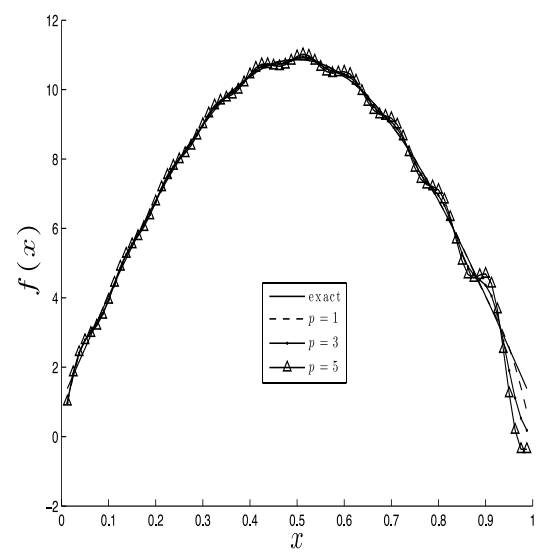

(b)

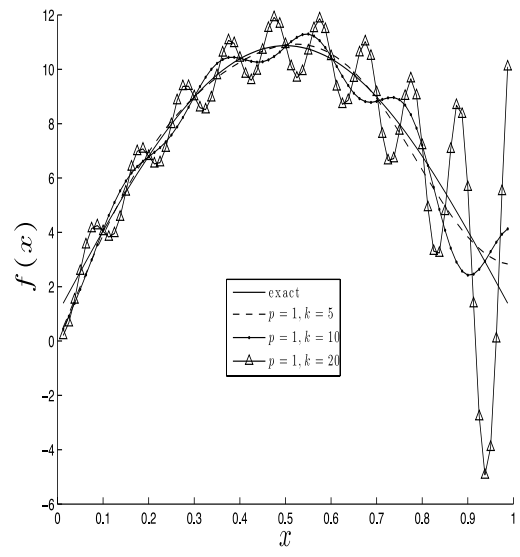

Figure 4-The exact solution (30) for $\boldsymbol{f}(\boldsymbol{x})$ in comparison with the numerical solution (23), (a) for various $\mathbf{p} \% \in\{\mathbf{1}, \mathbf{3}, \mathbf{5}\} \%$, and $\boldsymbol{K}=\mathbf{2 0}$ when Dirichlet boundary condition (9) is applied, (b) for various $\boldsymbol{K} \in\{\mathbf{5}, \mathbf{1 0}, \mathbf{2 0}\}$ and $\boldsymbol{p} \%=\mathbf{1} \%$ when mixed boundary condition (10) is used, with noisy data.

(a)

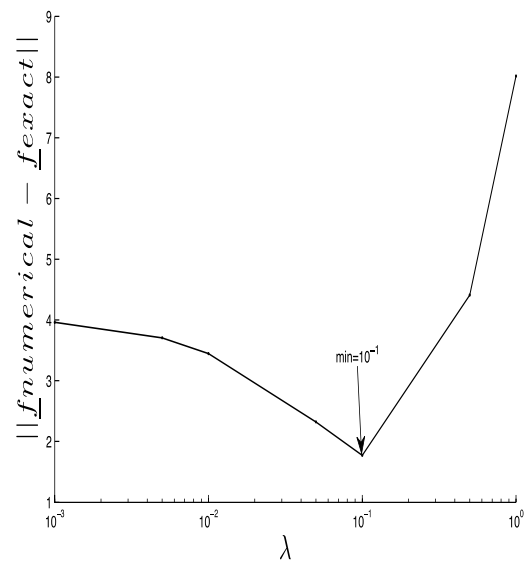

(b)

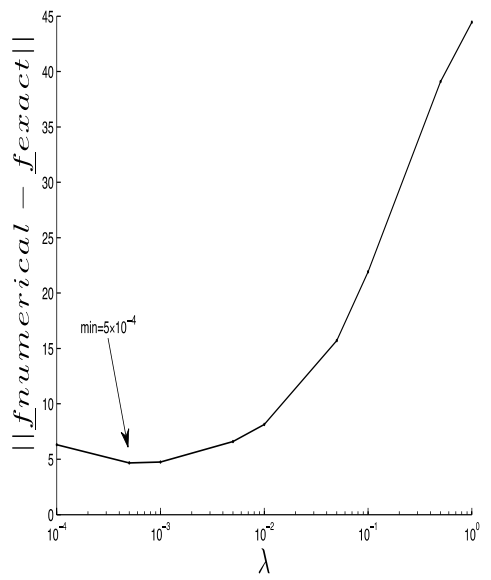

Figure 5-The accuracy error $\left\|f_{\text {numerical }}-f_{\text {exact }}\right\|$, as a function of $\lambda$, for $K=20$ and (a) $p \%=$ $5 \%$ noise, Dirichlet boundary condition $(9)$, (b) $p \%=1 \%$ noise, mixed boundary condition (10). 
(a)

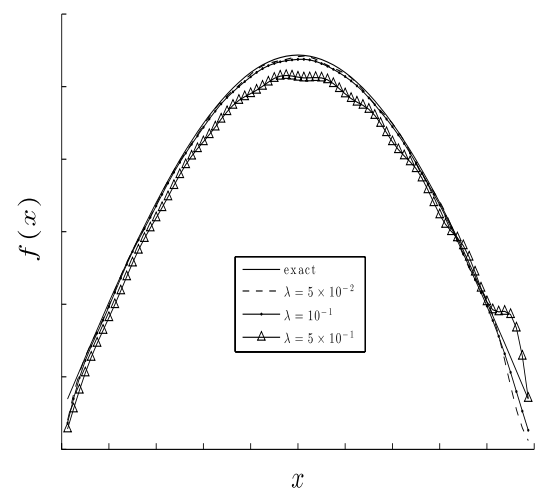

(b)

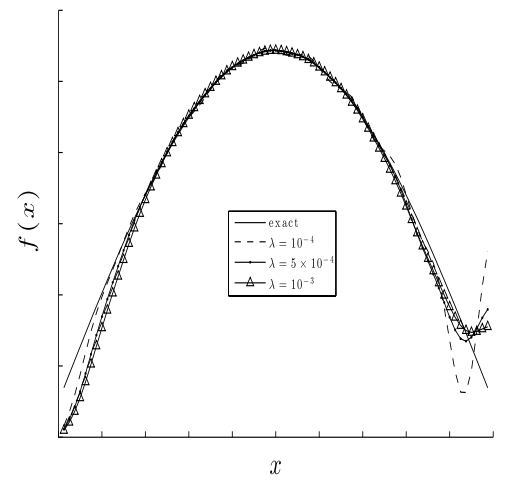

Figure 6-The exact solution (30) for $f(x)$ in comparison with the numerical (23), for $\mathrm{K}=20$, (a) $p \%=5 \%$ noise and regularization parameters $\lambda \in\left\{5 \times 10^{-2}, 10^{-1}, 5 \times 10^{-I}\right\}$, Dirichlet boundary condition (9) are applied, (b) $p \%=1 \%$ noise, and regularization parameters $\lambda \in\left\{10^{-4}, 5 \times\right.$ $\left.10^{-4}, 10^{-3}\right\}$, mixed boundary condition (10).

\section{Conclusions}

In this paper, we apply the one end data of Dirichlet or mixed boundary condition as additional data. Splitting the wave equation in two parts, first part was direct problem part when we solved it by (FDM), and second part was inverse problem part, in this part using separation of variables [3]. The problem is ill-posed, since adding a small noise in extra data causes an unstable force. In order to deal with this unsuitability, we employed the Tikhonov regularization method with minimum errors for selecting a good parameter for regularization. For future work, we plan to use $u(x, T)$ as additional information.

\section{References}

1. Hussein, S. O. and Lesnic, D. 2014. Determination of a space-dependent source function in the one- dimensional wave equation. Electronic Journal of Boundary Elements, 12: 1-26.

2. Hussein, S. O. 2016. January 1). Inverse force problems for the wave equation. Leeds, University Of Leeds, UK. "PhD Thesis"

3. Hussein, S. O. and Lesnic, D. 2016. Determination of forcing functions in the wave equation. Part I: the space-dependent case. Journal of Engineering Mathematics, 96: 115-133.

4. Huanga, C.-H. 2001. An inverse non-linear force vibration problem of estimating the external forces in a damped system with time-dependent system parameters. J Sound Vib, 242: 749-765.

5. Hussein, S. O. 2019. Splitting the one-dimensional wave equation. Part I: Solving by finitedifference method and separation variables. Baghdad Science Journal, (Under Reviews).

6. Cannon, J. R. and Dunninger, D. R. 1970. Determination of an unknown forcing function in a hyperbolic equation from overspecified data. Annali di Matematica Pura ed Applicata , 1: 49-62.

7. Colton, D. and Kress, R. 2013. Inverse Acoustic and Electromagnetic Scattering Theory. 3rd edn. New York: Springer Verlag.

8. Morse, P. M. and Feshbach, H. 1953. Methods of Theoretical Physics. New York: McGraw-Hill. 